\title{
Modalidades de innovación y construcción de capacidad tecnológica endógena de economías en desarrollo: los catalizadores en Brasil, Colombia y México, 1955-2009*
}

\author{
Henry Alfonso Mora Holguín** y Manuel Soria López ***
}

\section{RESUMEN}

Las industrias petroleras de Brasil, Colombia y México han desarrollado capacidad doméstica de aprendizaje, producción y acumulación de conocimiento tecnológico. Los cambios institucionales y las estrategias tecnológicas iniciadas en América Latina en los años noventa marcaron las diferencias en el desempeño económico de la industria petrolera de cada país. Estas tres naciones no han sido simples receptores de tecnología exógena. En distinto grado, desarrollaron procesos endógenos de aprendizaje y capacidad tecnológica, cuya modalidad de innovación depende del grado endógeno alcanzado para diseñar, producir y comercializar catalizadores para refinar combustibles del petróleo. Brasil, durante las últimas tres décadas, construyó, desarrolló y acumuló capacidad tecnológica exitosamente. En los diez años recientes, Colombia construyó y desarrolló capacidad tecnológica doméstica de forma marginal mediante procesos de aprendizaje ligados a empresas extranjeras. En México, la capacidad acumulada durante tres décadas para producir conocimiento tecnológico sobre catalizadores acusa deterioro.

Palabras clave: innovación endógena, capacidad tecnológica, país en desarrollo, industria del petróleo, catalizadores.

Clasificación JEL: O, O3, O31.

\section{AbSTRACT}

The oil industry of Brazil, Colombia and Mexico has developed domestic capability for learning, producing and accumulating technological knowledge. Institutional change and technological strategies initiated during the nineties in Latin America marked the differences of economic performance of the oil industry in each country. These three countries have not been simple receivers of exogenous technology. Each one of them has developed in a different degree endogenous processes of learning and technological capability in which the modality of innovation depends on the endogenous degree reached for designing, producing and selling catalyzers that refine oil into fuels. Brazil during the past three decades has built, developed and accumulated technological capability successfully. Colombia in the last ten years has also built and develops in a marginal degree domestic technological capability through processes of learning linked to foreign firms. In México during the past three decades the accumulated capability for producing technological knowledge on catalyzers has deteriorated.

Keywords: endogenous innovation, technological capability, developing country, oil industry, catalyzers.

JEL classification: $\mathrm{O}, \mathrm{O} 3, \mathrm{O} 31$.

* Fecha de recepción: 16/10/2011. Fecha de aprobación final: 01/08/2012.

** Maestro en Economía y Gestión de la Innovación. Investigador del Área de Innovación en el Observatorio Colombiano de Ciencia y Tecnología. Correo electrónico: hamorah@unal.edu.co.

*** Profesor-investigador del Departamento de Producción Económica, del Posgrado en Economía y Gestión de la Innovación, y del Área de Economía Industrial e Innovación, UAM-Xochimilco. Correo electrónico: msoria@correo.xoc.uam.mx. 


\section{INTRODUCCIÓN}

El desarrollo de una economía supone cambiar la estructura productiva y construir nuevos mercados, crear nuevas capacidades tecnológicas y transformar las instituciones de la sociedad. Es un proceso de transformación estructural de la interdependencia económica, tecnológica e institucional a lo largo de fases de construcción y destrucción de activos productivos y dispositivos distributivos en condiciones de incertidumbre, información imperfecta y fallas de mercado, dada una dinámica evolucionista (Kuznets, 1966; Abramovitz, 1956; Nelson y Winter, 1982; Katz, 2008).

En América Latina, esta transformación ha implicado un cambio estructural que retorna a las ventajas comparativas "naturales" en el marco de las nuevas tecnologías (Katz, 2008). Por ende, si se considera el desarrollo económico como fruto de la coevolución de fuerzas económicas, institucionales y tecnológicas, lograr capacidad tecnológica endógena implica aprendizaje, generación y acumulación de conocimiento tecnológico en el largo plazo (Lundvall, 1992), para dar paso a fenómenos de maduración de las fuerzas productivas y los mercados (Katz, 2008).

El objetivo es analizar cómo se ha desarrollado capacidad tecnológica endógena en materia de catalizadores para refinar combustibles en la industria petrolera de Brasil, Colombia y México. La pregunta es, entonces, ¿cómo explicar la evolución reciente en el desarrollo de capacidad tecnológica endógena de estos países? La proposición central es que la adquisición de capacidad tecnológica de la industria de refinados del petróleo en países selectos de América Latina ha seguido tres modalidades de innovación: a) tecnología exógena, b) tecnología exógena/endógena y c) tecnología endógena. Esto depende de la estrategia de largo plazo y de que determinados cambios institucionales y económicos detonen el desarrollo de procesos de aprendizaje y de acumulación de tecnología para la mejora del desempeño de los sistemas de producción. En general, el análisis de la evidencia indica un significativo avance de Brasil, un avance moderado de Colombia y un repliegue en México.

Este texto se desarrolla en cuatro secciones. Primero se explican las modalidades de innovación. La segunda compara tecnológica, económica e institucionalmente los casos de Brasil, Colombia y México. La tercera analiza la evolución de la capacidad tecnológica de cada país en términos de las tres modalidades de innovación. La cuarta sección despliega los hallazgos y reflexiones finales. 


\section{CONSIDERACIONES SOBRE LAS MODALIDADES DE INNOVACIÓN QUE CONTRIBUYEN A CONSTRUIR CAPACIDAD TECNOLÓGICA ENDÓGENA DE ECONOMÍAS EN DESARROLLO}

En el actual mercado global, es dentro de las empresas nacionales donde se construyen las capacidades tecnológicas; el estudio de ellas aporta elementos para comprender el origen de diferencias en productividad y calidad entre las empresas de determinadas industrias de países desarrollados y países en desarrollo. Tales diferencias desempeñan un papel central en la teoría del proceso evolutivo, que destaca la trascendencia del aprendizaje y la acumulación de conocimiento tecnológico para generar cambio técnico en los sistemas de producción (Nelson y Winter, 1982; Bell, 1984; Dosi, 1988; Bell y Pavitt, 1995). Los factores decisivos identificados en la construcción de capacidades tecnológicas a nivel de empresa en países en desarrollo son tanto internos -financiamiento de ID, desarrollo de nuevas habilidades, capacidad de absorción de nuevas tecnologías de producción, acumulación de conocimiento-, como externos - competencia en el mercado, cambio tecnológico, nueva inversión y desarrollo de empresas (Katz, 1976; Dahlman y Westphal, 1982; Teitel, 1984; Fransman, 1986; Vernon, 1989; Herbert-Copley, 1990; Rath, 1990; Lall, 1991; Torres, 2006; Dosi, 1988; Bell y Pavitt, 1995; Katz, 2007).

En general, cuando una empresa organiza el aprendizaje, acumula capacidad tecnológica. Aprender implica que los conocimientos tecnológicos adquiridos por la empresa sean absorbidos y contribuyan a construir capacidades tecnológicas muy específicas (Figueiredo, 2002; Torres, 2006). La capacidad de absorción supone crear nuevo conocimiento con base en la habilidad de la empresa para asimilar y explotar la información existente en su entorno (Cohen y Levinthal, 1989). El desarrollo de capacidades tecnológicas se refleja en la “endogenización" (es decir, la apropiación y adaptación a sus propias condiciones y para sus propios fines) de los conocimientos adquiridos por la empresa, a partir de diferentes fuentes tanto internas como externas, en los procesos de aprendizaje, la formación de recursos humanos y los productos de la ID (Garay, 1998). En una empresa, estas habilidades forman el sustrato para absorber tecnologías compradas y/o imitadas de otras empresas, lo que determina así su eficacia y eficiencia al explotar y mejorar tecnologías. Por ello, es probable que al comparar el uso de la misma tecnología en empresas de un país en desarrollo y de un país desarrollado, sea notoria la existencia de deficiencias en estas habilidades que imponen limitaciones a la fabricación del producto (Lall, 1993). 
La evidencia parece indicar que los países en desarrollo tienden a concentrar sus esfuerzos de búsqueda tecnológica en las actividades de investigación aplicada, es decir, al final del espectro, ya que para la investigación básica se necesitan más recursos (Katz, 1987). Sin embargo, esto no se aplica a todas las industrias de los países en desarrollo. En la de refinación del petróleo, el tipo de investigación que más se realiza es básica. El esfuerzo en investigación aplicada es menor porque se carece de los recursos y equipos industriales necesarios para escalar tecnológicamente las innovaciones. A diferencia de los países desarrollados, donde el cambio tecnológico se orienta en gran parte a reducir costos, en América Latina este proceso se ha caracterizado por adaptar tecnologías foráneas al mercado y a la cadena de suministro locales. Entre otros aspectos que generan diferencias al momento del aprendizaje entre empresas de países desarrollados y países en desarrollo, resaltan: el tamaño de la empresa, el sector (público, privado o social), la organización y concentración del mercado, el volumen y calidad de los recursos humanos especializados, y las políticas comerciales de protección y apertura (Teitel, 1984). En este contexto, para los países en desarrollo, la construcción de capacidades tecnológicas endógenas implica necesariamente la participación de agentes externos de países desarrollados. En efecto, el conocimiento proveniente del exterior viene incorporado en los especialistas, los equipos importados, las patentes, los manuales de instrucciones, las licencias y los contratos de asistencia técnica, mientras que el conocimiento tecnológico interno se encuentra en las actividades de ID propias de la empresa y sus agentes, entre otras fuentes.

Aunque es históricamente cierto que el cambio tecnológico se ha dado de manera más constante y rápida en los países desarrollados, también lo es que los países en desarrollo no han sido simples receptores pasivos de nueva tecnología. Diferentes estudios muestran cómo empresas manufactureras selectas de esos países participan activamente en el dominio, adaptación y creación de tecnología (Herbert-Copley, 1990). En efecto, como son las empresas en lo individual y no los sectores industriales quienes aprenden, parecería correcto pensar que las compañías latinoamericanas no llevan a cabo esfuerzos tecnológicos locales en sus tareas productivas. En la realidad sí se mejoran productos y procesos, se desarrollan nuevos modelos de organización de la producción, se crean y afianzan departamentos de ingeniería de todo tipo, se desarrollan proveedores y surgen, como subproducto del crecimiento, sinergias y externalidades de gran significación (Katz, 1976 y 2007). Aunque resulta claro que ello ocurre en una escala menor, como un resultado lateral de la producción y no como parte de una 
estrategia formal planeada o de políticas públicas diseñadas ex profeso. En efecto, existen compañías latinoamericanas que sí han creado procesos de aprendizaje a través de los cuales fueron adquiriendo ciertas habilidades venidas de los países desarrollados para luego construir su propia capacidad tecnológica en industrias particulares. ${ }^{1}$

En el periodo de la industrialización sustitutiva, este proceso era de carácter "endodirigido" en la medida en que el Estado dedicó, a través de las políticas industrial y arancelaria, sus mayores esfuerzos a consolidar un mercado interno, hasta que dicho modelo dio de sí al final de los setenta.

La transición de este modelo a uno de economía global y comercio internacional implicó construir y destruir capacidades tecnológicas específicas. Éste fue un periodo de aprendizaje en el que la mayoría de las compañías latinoamericanas estuvieron orientadas a los recursos naturales o a los productos de tecnologías maduras.

En suma, el vínculo entre las empresas de los países desarrollados y las empresas latinoamericanas ha sido crucial para aumentar el grado endógeno de la capacidad tecnológica desarrollada al incorporar la capacidad del aprendizaje en las empresas nacionales de los países en desarrollo (Katz, 2007).

Considerando la importancia actual del comercio global y las nuevas tecnologías, atribuimos al concepto "endógeno" una doble connotación. Primera, la relacionada al aprendizaje por imitación de las empresas nacionales de países en desarrollo de las tecnologías de países desarrollados. Segunda, el aprendizaje endógeno por la práctica acumulado históricamente en el sector económico.

\section{Metodología para medir la capacidad tecnológica entre países}

Tres son las dimensiones de la estructura conceptual. Primero, los factores institucionales de un nuevo arreglo que impulsan cambios en las organizaciones industriales. Segundo, el desarrollo de capacidad tecnológica, en especial, el aprendizaje tecnológico, la producción de conocimiento tecnológico y la acumulación tecnológica. Tercero, el sistema productivo al cual se incorpora la innovación tecnológica para cambiar el desempeño productivo y la composición del comercio exterior.

La metodología para obtener datos relevantes de la evidencia empírica se inspira en la perspectiva evolutiva de la economía de la innovación (Nelson

\footnotetext{
${ }^{1}$ Por ejemplo, la farmacéutica en México, con Syntex, Probiomed, Laboratorios Silanes, etcétera.
} 
y Winter, 1982; Dosi, 1981; Bell y Pavitt, 1995). Para medir la capacidad tecnológica de la economía de un país dado, se retoma la propuesta de Nelson (1981), así como las consideraciones de Lall (1993) para la economía de un país en desarrollo. La medición de capacidad tecnológica endógena se agrupa entonces en tres ámbitos: 1) Los recursos humanos que poseen el conocimiento tecnológico y las habilidades adquiridas por la experiencia (capital humano). 2) El esfuerzo tecnológico expresado por el nivel de gasto en ID y por los resultados tecnológicos en patentes otorgadas e innovaciones exitosas. 3) Finalmente, la inversión en la infraestructura de activos físicos básicos como instalaciones, maquinaria, equipos, materiales, etcétera. Para medir las capacidades tecnológicas en el sector petrolero de los países analizados en relación al desarrollo tecnológico y la producción industrial de catalizadores para refinar combustibles del petróleo, se retomó la propuesta de análisis llamada de la "triada innovadora" o "red de innovación" en materia de catalizadores del Instituto Mexicano del Petróleo (IMP), Petróleos Mexicanos (Pemex) y las empresas extranjeras (Aboites et al., 2004; Soria, 2004).

En este caso comparativo entre países -Brasil, Colombia y México-, los indicadores específicos serán los siguientes:

a) Variables económicas: PIB per cápita, producción de crudo, capacidad instalada de refinación y producción de petrolíferos.

b) Variables de capital humano: investigadores dedicados a actividades de ID como proporción de la población y variables de inversión en conocimiento y tecnología, como el gasto en ID tecnológica y en educación como proporción del PIB, así como infraestructura científica y tecnológica.

c) Variables de producción de conocimiento y tecnología: producción de conocimiento científico medida por artículos de investigación publicados y producción de conocimiento tecnológico medida por patentes solicitadas y publicadas.

El sentido interpretativo que tiene cada una de estas variables es como sigue: en el largo plazo, el crecimiento del producto final en cualquier economía de la fase actual del capitalismo depende de tres variables fundamentales: capital humano, producción de conocimiento e inversión en ID. Así, al comparar a las 
tres naciones latinoamericanas de este estudio en términos de la relación establecida entre dichas variables, un incremento en cualquiera de las variables independientes indica avance en la construcción y acumulación de capacidades tecnológicas. Al contrario, si cualquiera de las variables independientes muestra tendencia a la baja, esto indica que una economía nacional se aleja del objetivo de un desarrollo económico basado en el cambio tecnológico y la innovación.

\section{El esquema para el análisis}

Los principales supuestos en que se basa el esquema de análisis de la construcción de capacidades tecnológicas en materia de catalizadores para refinar combustibles del petróleo en Brasil, Colombia y México, son:

a) La innovación tecnológica es incremental (Pavitt, 1982): Los procesos de refinación de combustibles del petróleo sólo cambian en el largo plazo, por lo que el ajuste tecnológico para innovar en el corto y mediano plazo se da por los cambios que mejoran el diseño tecnológico del catalizador.

b) El aprendizaje tecnológico ocurre por imitación (Katz, 1976): En todos los casos latinoamericanos, el desarrollo de tecnología doméstica parte necesariamente de imitar tecnologías desarrolladas por países industrializados, por lo cual la relación de aprendizaje con empresas provenientes de éstos es indispensable.

c) El proceso de invención, innovación y producción de un catalizador (Aboites et al., 2004; Soria, 2004) ocurre a través de tres fases.

\section{a) Fase I: diseño tecnológico del catalizador}

Comienza con la demanda de la industria para refinar combustibles y finaliza con el diseño tecnológico de un catalizador ajustado a dicha demanda. Ésta depende a su vez de la composición química del petróleo crudo a refinar, la planta refinadora y la composición de la demanda final de combustibles. Incluye las actividades de análisis de los catalizadores disponibles en el mercado, así como el tipo de contrato para su desarrollo y producción, por licitación o asignación directa, a escala nacional o mundial. La parte nodal de esta fase es el proceso de ID tecnológica, mediante el cual se diseña el catalizador y que incluye tanto las actividades de investigación básica de materiales catalíticos, como la aplicada en laboratorio y la prueba del catalizador en la planta piloto. 


\section{b) Fase II: escalamiento industrial del catalizador}

Implica la existencia de empresas domésticas productoras de catalizadores. El proceso de escalamiento industrial de la tecnología significa retomar el diseño tecnológico desarrollado en la fase anterior e irlo probando y ajustando en términos de su capacidad tecnológica real para refinar de la forma esperada determinados volúmenes de petróleo en condiciones de una planta industrial. Si las pruebas de esta segunda fase son superadas, se procede a producir el primer lote del catalizador para la planta industrial donde será utilizado realmente.

\section{c) Fase III: comercialización del catalizador}

Empieza con la realización de la prueba industrial con el lote de la fase anterior. Si es exitosa, se producirán nuevos lotes del catalizador a un nivel de consumo industrial, tendrán lugar una serie de procedimientos de venta y serán necesarios los servicios de mantenimiento del catalizador en planta a lo largo de su vida útil. En esta fase se incluyen, asimismo, algunas mejoras incrementales, las cuales se realizan entre la producción de un lote y otro.

\section{d) Grado endógeno de la producción de catalizadores}

El concepto utilizado para identificar la construcción y desarrollo de capacidades tecnológicas en los países analizados es el de "grado endógeno" del proceso de producción de catalizadores antes descrito. En efecto, dependiendo de la capacidad tecnológica de la industria petrolera de cada país, el proceso de innovación tecnológica se hará de modo más o menos endógeno (Aboites, et al., 2004; Soria, 2004).

Conforme se alcanzan dichas fases se establecen modalidades de la innovación tecnológica, las cuales, a su vez, se encuentran estrechamente ligadas al nivel de desarrollo de cada país (gráfica 1):

Modalidad A: Empresas nacionales de una economía en desarrollo importan y transfieren del exterior casi la totalidad de la tecnología para operar un sistema productivo $x$ en una industria $z$.

Modalidad B: Empresas nacionales de la industria $z$ aprenden a producir diseños tecnológicos de ciertos insumos estratégicos imitando diseños de empresas extranjeras, aunque no cuentan con capacidad doméstica para su producción. 
Modalidad C: Empresas nacionales de la industria $z$ lograron desarrollar tanto capacidades de ID como sistemas de producción de insumos estratégicos para la industria $x$.

Gráfica 1. La innovación tecnológica de catalizadores para refinar combustibles

\begin{tabular}{|c|c|c|c|c|}
\hline $\begin{array}{c}\text { Fase de } \\
\text { innovación } \\
\text { Producción y } \\
\text { comercialización } \\
\text { doméstica } \\
\text { Escalamiento } \\
\text { industrial del } \\
\text { catalizador } \\
\text { Capacidad de ID } \\
\text { tecnológica en } \\
\text { catálisis } \\
\text { Sin capacidad de } \\
\text { ID tecnológica }\end{array}$ & $\begin{array}{l}\text { Países de menor } \\
\text { desarrollo }\end{array}$ & $\begin{array}{l}\text { Países en } \\
\text { desarrollo }\end{array}$ & $\begin{array}{c}\text { Países } \\
\text { desarrollados } \\
\text { Países } \\
\text { emergentes }\end{array}$ & \\
\hline & $\begin{array}{c}\text { A } \\
\text { (+) Exógena }\end{array}$ & B & $\begin{array}{c}c \\
\text { (+) Endógena }\end{array}$ & $\begin{array}{l}\text { Modalidad de } \\
\text { innovación }\end{array}$ \\
\hline
\end{tabular}

Fuente: Elaboración propia, a partir de Soria (2004) y Mora (2010).

En suma, conforme se alcanza la fase más alta -comercialización y producción del catalizador-, el proceso de construcción y desarrollo de capacidades tecnológicas se vuelve más endógeno y es característico de países desarrollados y algunos emergentes. Al contrario, a menor desarrollo relativo de los países casi no existen capacidades tecnológicas, por lo tanto, las innovaciones tecnológicas requeridas se transfieren casi plenamente por importación del producto final.

\section{EVOLUCIÓN DE LA CAPACIDAD TECNOLÓGICA ENDÓGENA PARA DISEÑAR Y PRODUCIR CATALIZADORES}

En el desarrollo económico y social de América Latina -desde la posguerra hasta la actualidad- (Katz, 2008), se distinguen tres fases:

1. Crecimiento "hacia adentro" liderado por el Estado ("endodirigido"), 1945-1979.

2. Turbulencia y desequilibrio macroeconómico, 1980-1989.

3. Tecnología, instituciones y regímenes competitivos en una economía abierta y desregulada, 1990-2011. 
En efecto, desde los años ochenta se observa un proceso de destrucción y creación en el desarrollo de capacidades sociales de organización de la producción, de nuevas instituciones y de tecnologías (Katz, 2008). El desenvolvimiento de las economías desde los años cincuenta bajo el liderazgo del Estado dio paso a un nuevo paradigma fundado en una economía abierta a la competencia externa, desregulada y privatizada, cuya base fue, y es todavía, la explotación de los recursos naturales. En este contexto histórico, entre las economías latinoamericanas, Brasil, Colombia y México en conjunto cuentan con una importante dotación de petróleo, aunque en particular cada una de sus industrias petroleras es diferente en tamaño y nivel de desarrollo. El propósito de esta sección es determinar el grado endógeno de la capacidad tecnológica para producir catalizadores para refinar combustibles del petróleo.

\section{Naturaleza de las compañías petroleras de los países analizados}

Una característica común a los tres países analizados es la intervención del Estado en el proceso de formación y desarrollo de la industria del petróleo. Primero, hacia finales del siglo XIX y principios del pasado, un conjunto de Estados latinoamericanos entregaron sus campos petroleros a las empresas extranjeras y éstas crearon la primera estructura industrial para su explotación. Luego, hacia mediados del siglo XX vino un proceso de recuperación del petróleo en poder de las empresas multinacionales mediante expropiaciones estatales. Esta fase se caracterizó por la creación de una sola compañía petrolera nacional y monopólica. Sin embargo, actualmente y con el cambio institucional, ${ }^{2}$ en algunos países se ha modificado de manera importante la industria petrolera, como veremos.

En este contexto, la creación y desarrollo de los centros de ID nacionales para esta industria en los tres países latinoamericanos se inició en diferentes momentos, con resultados distintos en el desarrollo de capacidad tecnológica y la construcción de catalizadores. Al principio, algunos de estos centros de investigación se fundaron, por ejemplo, debido a la necesidad de formar recursos hu-

\footnotetext{
${ }^{2}$ Las instituciones y el cambio institucional son un fenómeno complejo que requiere para su análisis de la contribución de distintos paradigmas (Campbell y Pedersen, 2001). No obstante, para efectos prácticos, en este texto se considera a las instituciones económicas sujetas a las reglas de comportamiento que siguen las organizaciones y los agentes económicos (North, 1993). Cuando cambian estas reglas y modifican el comportamiento de los agentes involucrados, entonces, el cambio institucional tiene lugar, aunque éste puede ser o no el esperado y no implica necesariamente la modificación de los arreglos institucionales en su conjunto.
} 
manos especializados (Brasil, en 1954). Otros, para determinar si los precios que pagaban por insumos estratégicos eran los correctos (México, en 1965). Con el tiempo, aunque estos centros evolucionaron conforme a modalidades específicas, en todos los casos se incorporaron a las actividades de investigación y desarrollo, búsqueda de soluciones tecnológicas, servicios técnicos, capacitación y comercialización de productos desarrollados.

La compañía petrolera nacional Petróleos Brasileños (Petrobras) se constituyó en octubre de 1953, reemplazando en dichas actividades al denominado Consejo Nacional del Petróleo (CNP), el cual a partir de entonces se transformó en una entidad pública fiscalizadora de la industria. Desde su creación, Petrobras mantuvo el monopolio sobre la exploración, la explotación, el transporte y la refinación de petróleo en Brasil a escala mayorista, ya que la comercialización de los productos al por menor en las estaciones de servicio estuvo excluida del monopolio desde un principio (Petrobras, 2010).

A partir de la apertura comercial global de los noventa, el gobierno federal de Brasil consiguió impulsar el cambio estructural en el sector energético al retirarle a Petrobras el monopolio (1995), permitiendo el ingreso de empresas extranjeras para explorar y explotar yacimientos en suelo brasileño (1997), colocando acciones en el mercado internacional (2000) y creando la Agencia Nacional del Petróleo (ANP) como reguladora y fiscalizadora de la industria (1995). En suma, la disminución en la intervención del Estado, el refinamiento del control fiscal para efectos del bienestar nacional, la apertura a la inversión de capital extranjero y la entrada de compañías internacionales del ramo resultaron en un crecimiento importante de la industria petrolera en Brasil en términos de sus reservas probadas, capacidad de extracción y refinación de petróleo.

Como parte de la estrategia para la industria del petróleo durante el monopolio de Petrobras, se creó en 1955 el Centro de Perfeccionamiento e Investigaciones de Petróleo, hoy Centro de Pesquisas Leopoldo Américo Miguez de Mello (Cenpes). Originalmente, estuvo dedicado a formar capital humano y luego, entre 1963 y 1975, profundizó en la actividad de ID tecnológica dirigida a la industria del petróleo mediante la reestructuración del centro y la creación de nuevas divisiones de ID especializadas. La división de catalizadores no se creó hasta 1985, con el objeto de dar soporte al desarrollo de productos y a la producción de catalizadores de craqueo catalítico ${ }^{3}$ en lecho fluido (FCC, por sus siglas en in-

\footnotetext{
${ }^{3}$ Proceso químico por el cual se quiebran moléculas de un compuesto produciendo así compuestos más simples.
} 
glés) para refinar gasolina en Brasil. En la última década, Petrobras ha destinado el 1\% de sus ingresos brutos al Cenpes (2009), con lo que éste alcanza el nivel más alto de gasto en ID en América Latina y montos de inversión similares a los de las empresas globales de países desarrollados (Petrobras, 2010).

A mediados del siglo XX, en 1951 específicamente, se logró la creación de la Empresa Colombiana de Petróleos (Ecopetrol) al expropiarse los activos de la Tropical Oil Company (1921). Al inicio de la década actual (2003), el cambio estructural en la industria petrolera colombiana consistió en la reorganización de Ecopetrol como una sociedad de economía mixta, copropiedad del Estado y del sector privado nacional y/o extranjero. A la par, se creó la Agencia Nacional de Hidrocarburos (ANH), cuyo objeto ha sido fiscalizar el petróleo en tanto recurso natural de Colombia. El resultado de esta reforma institucional en el actual mercado petrolero de Colombia ha sido la participación de diferentes compañías petroleras, entre las cuales Ecopetrol es la dominante con la cuota más alta (58\%) del mercado nacional (Ecopetrol, 2010 a). El centro de ID propiedad de Ecopetrol (1985) es el Instituto Colombiano del Petróleo (ICP). Éste contribuye a desarrollar tecnología y conocimiento para elevar la productividad en los procesos de búsqueda, producción, transporte y refinación de hidrocarburos. Desde su creación, el ICP ha desarrollado más de 115 productos tecnológicos relevantes para su crecimiento que se materializaron en métodos, procesos, productos, equipos, herramientas y software. El área de catálisis se incluyó desde el inicio del centro, siendo su principal objetivo evaluar y seleccionar catalizadores de FCC para refinar gasolina (Ecopetrol, 2010a).

En México, la industria petrolera nacional se fundó con la expropiación de las compañías extranjeras en 1938 y la creación de Petróleos Mexicanos (Pemex) como empresa monopólica estatal del sector de los hidrocarburos en beneficio del país. Al inicio de la apertura comercial (1992), la Ley Orgánica de Pemex se modificó, aunque sólo para efectos incrementales en sus procesos organizativos, ${ }^{4}$ más no en términos de dinamizar la estructura de la competencia en el mercado o de la inversión privada nacional o extranjera en la industria o del desarrollo de capacidad tecnológica endógena (Pemex, s.f.a).

Otra diferencia, respecto del Cenpes de Brasil o del ICP de Colombia, es que en México el Instituto Mexicano del Petróleo (IMP) se creó como un organismo descentralizado independiente legalmente de Pemex, cuya función ha sido

\footnotetext{
${ }^{4}$ Corporativo Pemex: Pemex Exploración y Producción, Pemex Refinación, Pemex Gas y Petroquímica Básica, y Pemex Petroquímica.
} 
proporcionarle a esta empresa servicios tecnológicos y actividades de ID tecnológica (Soria, 2004). El área de catálisis del IMP se creó casi desde el inicio (1967) y ha logrado construir hasta hace algunos años capacidades tecnológicas domésticas para el diseño de catalizadores de FCC, hidrodesulfuración, reformación, etcétera, en sociedad con empresas extranjeras, para ser utilizados en las plantas de Pemex. Sin embargo, en la década reciente hubo una sensible declinación en la inversión en ID tanto por parte del IMP como de Pemex y un incremento sustancial en las actividades de servicios tecnológicos (CONACYT, s.f.).

La naturaleza de las compañías petroleras nacionales y sus centros de investigación se perfila como una de las principales diferencias institucionales entre los países analizados. En efecto, los cambios institucionales dirigidos al sector petrolero en Brasil y Colombia no sólo lograron la incorporación de inversionistas privados, sino también de canales de aprendizaje tecnológico, debido a que la ID es parte de la estrategia general de Petrobras o de Ecopetrol. Caso contrario el de México, en donde no ha habido cambio institucional significativo en la organización industrial del sector, además que en la relación institucional de Pemex con el IMP no siempre se comparte la misma estrategia tecnológica.

En suma, la experiencia de estos países se encuadra en las fases de desarrollo económico y social de las economías latinoamericanas (Katz, 2008). La etapa de "crecimiento hacia adentro" liderado por el Estado da cuenta de la creación de las compañías petroleras nacionales (1938-1953) y de los centros de ID de la industria (1955-1985).

Durante la etapa de "turbulencia y desequilibrio macroeconómico" (1980-1990), la crisis empujó a diversos países de América Latina a realizar determinados cambios estructurales y regulatorios de la industria basada en los recursos naturales y de sus economías. En efecto, para la tercera etapa, "tecnología, instituciones y regímenes competitivos en economía abierta y desregulada" (1991-2011), si bien la tendencia del cambio institucional iniciada en Brasil en los noventa y seguida en Colombia se orientó a abolir el monopolio del Estado en la industria del petróleo, a la apertura hacia la inversión extranjera y a la entrada de nuevos competidores en la industria local (1995-2001), en México, a pesar de su incorporación al TLCAN (1994) y la OMC (1995), no hubo una propuesta de cambio institucional en ese mismo sentido. Asimismo, cabe señalar que el cambio institucional en Brasil y Colombia logró incorporar nuevas inversiones, tecnologías y agentes competitivos, al final, el acomodo industrial mantiene todavía como empresas dominantes a las que antes fueron ciento por ciento monopolios estatales. 


\section{Industria petrolera y capacidad de refinación de crudo en América Latina}

A nivel del sector de hidrocarburos, los países de la región latinoamericana más importantes han sido Venezuela y México, aunque recientemente ha crecido la participación de Brasil en la producción de petróleo y de Bolivia en la de gas. Los datos de producción de crudo indican que México ocupa el séptimo lugar mundial y el primero a nivel latinoamericano $(3001 \mathrm{mbd}$, millones de barriles diarios), mientras que Brasil es el noveno productor mundial y el segundo en América Latina (2571 mbd) y Venezuela ocupa el puesto once del mundo y el tercero en la región (2472 mbd). Les siguen Argentina, cuarto lugar latinoamericano (796 mbd), y Colombia en el quinto (680 mbd) (EIA, s.f.).

El principal consumidor de petrolíferos en la región entre 2008 y 2009 (EIA, s.f.) fue México con la mayor demanda de refinados (2485 mbd), de los cuales sólo destiló dos terceras partes $(1540 \mathrm{mbd})$ y compró en el exterior la tercera parte restante. Brasil le sigue con una demanda cercana (2 $128 \mathrm{mbd})$, la cual satisfizo destilando la mayor porción (1908 mbd) e importó sólo poco más de la décima parte de sus requerimientos totales. A su vez, Venezuela tuvo una demanda de refinados menor que los anteriores (1282 mdb), seguida por Argentina (626 $\mathrm{mdb}$ ) y Colombia (286 mdb).

Dentro de los sistemas de refinación de petróleo de estos países, la capacidad para producir gasolina mediante el método de FCC fue mayor en Brasil (505 mbd), seguido de México (381 mbd), Venezuela (232 mbd), Argentina (141 $\mathrm{mbd}$ ) y Colombia (90 mbd). En cuanto al proceso de reformación catalítica, el país con la mayor capacidad productiva fue México (279 mbd), y luego Argentina (57 mbd), Venezuela (50 mbd) y Brasil (24 mbd).

Finalmente, respecto a la capacidad para refinar petróleo a través de $\mathrm{cra}$ queo térmico, Colombia fue el país latinoamericano con mayor capacidad productiva (52 mbd), seguido por Argentina (38 mbd).

Brasil y Venezuela son los países que han mostrado en la región el mayor dinamismo en cuanto al incremento de capacidad de refinación entre 1996 y 2006. Brasil aumentó en $31 \%$-el mayor crecimiento en la región latinoamericana- su capacidad de producir refinados al pasar de $1481 \mathrm{mbd}$ a $1941 \mathrm{mbd}$. Por su parte, Venezuela registró un crecimiento de $11 \%$ al añadir $128 \mathrm{mbd}$ a su capacidad de refinación, quedando ésta en $1289 \mathrm{mbd}$. En ambos casos, estos incrementos son el resultado de estrategias de ambos países que buscan no sólo aumentar sus actuales capacidades internas de refinación sino también convertirse en exportadores de petrolíferos (Sener, 2007 b). 
De las tres compañías analizadas, Petrobras fue la que mayor inversión canalizó en 2008, por un monto de $25 \%$ de sus ingresos, es decir, 29 mmd (miles de millones de dólares) (Petrobras, 2010). Le siguió Ecopetrol, que invirtió el $23 \%$ de sus ingresos, es decir, 4.6 mmd (Ecopetrol, 2008). En tercer lugar se encuentra Pemex, con una inversión de $15 \%$ de sus ingresos, alrededor de $18 \mathrm{mmd}$ (Pemex, s.f.b). Esto indica la importancia que tiene para Petrobras invertir en nuevas tecnologías, aumentar su capacidad instalada y realizar proyectos que le permitan mejorar sus capacidades. Particularmente de las inversiones en el sector de refinados, Pemex fue la compañía que más invirtió en 2008, con $6.8 \mathrm{mmd}$ (Pemex, s.f.b), seguido de Petrobras, con 5.5 mmd (Petrobras, 2009 a) y Ecopetrol, con 476 md (millones de dólares) (Ecopetrol, 2010b).

La cantidad de catalizadores que consume cada uno de estos países al refinar el petróleo que producen no se conoce con exactitud, aunque en general entre mayor es la producción de petrolíferos, mayor el consumo de catalizadores. Sin embargo, si se sabe cuál es la capacidad de refinación y el tipo de crudo procesado, se puede tener una idea de los tipos de catalizadores utilizados para refinar la clase de crudo producido.

La producción de refinados del petróleo en Brasil, Colombia y México se concentra en combustibles como gasolina, diesel y combustóleo, mientras que los principales catalizadores son los de craqueo catalítico en lecho fluido (FCC) e hidrotratamiento (HT) (Mora, 2010). En Brasil, los catalizadores más demandados por las refinerías para procesar crudos ligeros y pesados son los de FCC (95\%), seguidos por los catalizadores de HT para refinar crudos pesados y extrapesados (5\%). En Colombia, los catalizadores más demandados por las refinerías son los de FCC (50\%) para procesar crudos superligeros y ligeros, seguidos por los de HT para procesar pesados (50\%). A su vez, en México la principal demanda es por catalizadores para HT de crudos pesados y extrapesados $(70 \%)$, seguidos por los de FCC para refinar los demás petróleos crudos extraídos (30\%).

Los principales indicadores de producción, consumo y balanza comercial de petróleo crudo y refinado en los tres países (cuadro 1) muestran lo siguiente: la producción anual promedio de crudo se incrementó en los tres países -Brasil, 104\%; México, 11\%, y Colombia, 6\%-. En efecto, si bien México continuó como el mayor productor, Brasil aumentó considerablemente su nivel de producción y Colombia ligeramente. En relación al consumo promedio de productos refinados, en los tres países hubo un incremento-Brasil, 53\%; Colombia, $5 \%$, y México, 6\%-. A su vez, con relación al crecimiento de la capacidad de refinar petróleo, el mayor cambio se registró de nuevo en Brasil (48.6\%), seguido 
de Colombia (6.7\%) y México (2.1\%). Finalmente, si bien el porcentaje de utilización de sus refinerías es similar entre los tres (82\% a 89\%), en México es más bajo que en Brasil y Colombia.

En suma, mientras Brasil aumentó significativamente su capacidad de consumir, producir y refinar petróleo, en Colombia ese cambio fue más moderado. Sin embargo, en México, siendo el mayor productor latinoamericano de petróleo, en dos décadas prácticamente no se fomentó el crecimiento de la industria de la refinación, se mantuvo su plataforma exportadora de crudo y aumentó moderadamente su consumo de refinados.

\section{Cuadro 1. Producción y consumo de crudo y refinados en la industria} petrolera de Brasil, Colombia y México, 1989-2009

(millones de barriles diarios, promedio anual por subperiodo)

\begin{tabular}{|l|rr|rc|cc|}
\hline \multirow{2}{*}{\multicolumn{1}{|c|}{ Indicador }} & \multicolumn{2}{|c}{ Brasil } & \multicolumn{2}{c}{ Colombia } & \multicolumn{2}{c|}{ México } \\
\cline { 2 - 8 } & $1989-1999$ & $2000-2009$ & $1989-1999$ & $2000-2009$ & $1989-1999$ & $2000-2009$ \\
\hline Producción de crudo & 978 & 2005 & 560 & 593 & 3194 & 3546 \\
\hline Consumo de petrolíferos & 1727 & 2248 & 246 & 274 & 1845 & 2043 \\
\hline Capacidad de refinación & 1407 & 1882 & 248 & 286 & 1525 & 1593 \\
\hline $\begin{array}{l}\text { Utilización de refinerías } \\
\text { (porcentaje) }\end{array}$ & \multicolumn{2}{|c|}{87.13} & & 89.39 & & 82.47 \\
\hline Balanza comercial de crudo & -540 & -152 & 277 & 245 & 1504 & 1912 \\
\hline Balanza comercial de refinados & -119 & -74 & 34 & 74 & -90 & -279 \\
\hline
\end{tabular}

Fuente: Elaboración propia con base en datos de Petrobras (s.f.), Ecopetrol (s.f.), Pemex (s.f.b) y EIA (s.f.).

Con relación al comercio exterior de petróleo en cada país, se observa que Brasil tiene una tendencia decreciente en sus importaciones de crudo, dado el crecimiento de la producción interna. Asimismo, el desarrollo de su industria de refinación se expresa en un déficit decreciente en la importación de refinados. Colombia, por su parte, mantiene una balanza positiva; si bien las exportaciones de crudo fueron menores, las de refinados tienen un superávit creciente. México, a su vez, muestra un comportamiento divergente al de estos dos países: no sólo aumentó el volumen exportado de petróleo crudo, sino que a la par aumentó su déficit comercial de refinados.

En resumen, Petrobras mostró capacidades crecientes para producir tanto petróleo crudo como refinado y se posicionó con la mayor capacidad instalada 
en refinerías de América Latina. Pemex se caracterizó por ser un exportador neto de petróleo crudo y el mayor importador de productos refinados de América Latina. Ecopetrol, a su vez, no aumentó significativamente su capacidad de refinación, aunque, al incrementar el volumen de crudo procesado en refinerías mantuvo una balanza comercial superavitaria. Así, en la década reciente, la industria del petróleo de Brasil tuvo el desarrollo industrial más dinámico, frente a uno moderado en Colombia y la franca desaceleración en México.

\section{Comparación de la construcción de capacidad tecnológica endógena}

Las capacidades tecnológicas de los países seleccionados se examinan en esta sección conforme a las tres fases del esquema de análisis ya delineado, exponiendo los principales indicadores sobre gasto en ID, infraestructura de ID y capital humano (cuadro 2), con los cuales cuentan para desarrollar catalizadores los centros de investigación de las respectivas compañías petroleras.

Cuadro 2. Gasto, infraestructura y capital humano para actividades de ID de catalizadores en Brasil, Colombia y México, 2008-2009

\begin{tabular}{|l|ccc|}
\hline \multicolumn{1}{|c|}{ Indicador } & $\begin{array}{c}\text { Cenpes } \\
\text { Brasil }\end{array}$ & $\begin{array}{c}\text { ICP } \\
\text { Colombia }\end{array}$ & $\begin{array}{c}\text { IMP } \\
\text { México }\end{array}$ \\
\hline $\begin{array}{l}\text { Gasto en ID, 2008 (porcentaje del ingreso de la } \\
\text { petrolera nacional) }\end{array}$ & 0.8 & 0.4 & 0.05 \\
\hline $\begin{array}{l}\text { Gasto en ID para catálisis, 2008 (porcentaje del } \\
\text { gasto total en ID) }\end{array}$ & 16 & 30 & 10 \\
\hline Plantas piloto para catálisis, 2008 (número) & 30 & 8 & 19 \\
\hline Investigadores del centro de ID, 2009 (número) & 2034 & 193 & 661 \\
\hline $\begin{array}{l}\text { Inventores con al menos una patente de } \\
\text { catálisis, 1975-2009 (número) }\end{array}$ & 121 & 26 & 103 \\
\hline $\begin{array}{l}\text { Inventores de catálisis / Total de investigadores } \\
\text { (porcentaje) }\end{array}$ & 5.9 & 13.5 & 15.6 \\
\hline
\end{tabular}

Fuente: Elaboración propia con base en datos de Ecopetrol (s.f.), Petrobras (s.f.), Pemex (s.f.b) e Instituto Mexicano del Petróleo (2008b).

\section{a) Diseño tecnológico del catalizador}

En relación con el gasto en investigación y desarrollo, en Brasil, el Cenpes obtuvo el $0.82 \%$ de los ingresos de Petrobras (2009b), mientras el ICP de Colombia ejerció como gasto el $0.4 \%$ de los ingresos de Ecopetrol (2009). En el caso de 
México, de los 62 md gastados en ID, el 29\% fue financiado directamente por el IMP y el restante $71 \%$ provino de Pemex (s.f.b). El total del gasto en ID como porcentaje de los ingresos de Pemex apenas representó el 0.05\%. En términos absolutos, Petrobras gastó en actividades de ID quince veces más de lo que gastó Pemex y once veces más de lo que gastó Ecopetrol. En el caso de la actividad de ID para catalizadores de cada empresa, la colombiana le destinó el 30\% de su gasto total en ID, mientras la brasileña le dedicó $16 \%$ y el IMP sólo $10 \%$.

En cuanto a la infraestructura y el capital humano, el Cenpes de Brasil tiene la mayor cantidad de plantas piloto (30) y de investigadores dedicados a actividades de ID (2034), así como la mayor cantidad de investigadores que han trabajado en patentes de catálisis (137). En segundo lugar se encuentra el IMP, con un nivel intermedio de plantas piloto (19), investigadores totales (661) e investigadores en catálisis (110). En tercer lugar queda el ICP, con el nivel más bajo de plantas piloto (6), investigadores totales (193) e investigadores en catálisis (26). Como se observa, la industria petrolera de Brasil presenta cifras más altas de infraestructura y capital humano que México y Colombia, lo cual refleja un proceso creciente en el desarrollo de capacidad tecnológica doméstica en Brasil.

En relación con la producción de conocimiento sobre catalizadores registrado en las oficinas nacionales de patentes de cada país (1985-2009), la evidencia para este periodo indica lo siguiente: En el Instituto Nacional da Propriedade Industrial (INPI) de Brasil se han registrado más de 400 solicitudes de patente sobre catalizadores. En el Instituto Mexicano de la Propiedad Industrial (IMPI) se presentaron 230 solicitudes y en la Superintendencia de Industria y Comercio (SIC) de Colombia se registraron 135 solicitudes de catalizadores. En Brasil, el 21\% de las patentes solicitadas fueron presentadas por empresas o centros de ID residentes, mientras que en México este indicador fue de $9 \% \mathrm{y}$ en Colombia alcanzó sólo el 7\%. En la última década, a los residentes mexicanos se les ha otorgado un total de 55 patentes de catalizadores, a los brasileños 30 y a los colombianos tres, lo cual muestra cómo en México la producción de conocimiento medida por patentes ha sido significativa en comparación con Brasil y Colombia, aunque no se ha plasmado en el desarrollo de capacidades de refinación.

En producción de conocimiento a nivel internacional, medida por patentes registradas en la United States Patent and Trademark Office (USPTO), este organismo ha otorgado en la última década 21 patentes sobre catalizadores para refinar petróleo a Petrobras. Por su parte, Pemex no aparece con patente alguna y el IMP sólo con cinco. Finalmente, en el mismo lapso, una sola patente le ha 
sido otorgada a Ecopetrol. Esta evidencia indica, entonces, cómo Petrobras es una empresa con proyección tecnológica internacional, a diferencia de Pemex, que no cuenta siquiera con patentes internacionales. Por su parte, la presencia del IMP y de Ecopetrol es marginal en la USPTO, lo cual indica que su actividad es sólo de alcance doméstico.

En un análisis de las patentes sobre catalizadores concedidas en los tres países (ante IMPI, INPI y SIC, de 1975 a 2000) que haga una comparación de datos entre los periodos 1975-1987 y 1988-2000, la evidencia sobre los residentes en cada país indica un crecimiento de las patentes sobre catalizadores en los tres países durante el segundo lapso: Brasil, 44\%; Colombia, 23\%, y México, 31\%. En particular, Petrobras y Ecopetrol incrementaron las patentes otorgadas durante el periodo $1988-2000$, la primera $43 \%$ y la segunda $25 \%$. Por su parte, Pemex no registró patente alguna, mientras el IMP tuvo un aumento moderado (10\%). Como se observa, la estrategia tecnológica de Pemex difiere de la adoptada por Petrobras y Ecopetrol, las cuales han ido incrementando su capacidad para producir conocimiento.

Sin embargo, en términos relativos, el IMP es la organización que mayor proporción de investigadores ha asignado a la tarea de inventar catalizadores. En efecto, si se considera el número de inventores que han participado en al menos una patente de catálisis registrada en las oficinas nacionales respectivas contaban como porcentaje del número total de investigadores con los que contaba cada centro de ID a 2009, se obtiene que México alcanza una proporción de 15.6\%, mientras Colombia un $13.5 \%$ y Brasil el 5.9\% (cuadro 2). Es decir, en términos relativos, la inversión en producción de conocimiento en México en las últimas cuatro décadas no ha sido menor, incluso ha sido más significativa, que en Brasil y Colombia. Sin embargo, la contribución del IMP al actual desempeño tecnológico de la industria de refinación se ha estancado, a diferencia de la actividad de ID en Brasil y Colombia.

\section{b) Escalamiento industrial del catalizador}

Esta fase del desarrollo tecnológico requiere de un conjunto de pruebas a diferentes escalas ordenadas progresivamente. En los tres países, los centros de investigación y sus recursos humanos cuentan con la capacidad tecnológica necesaria para formular nuevos catalizadores en sus áreas de especialidad, probarlos en sus laboratorios y luego en las plantas piloto diseñadas para tal propósito. A partir de los resultados de estas pruebas se hacen las mejoras necesarias. Una vez 
que se tienen los resultados esperados, se procede a organizar la prueba industrial del catalizador. Como esta prueba se realiza en una refinería como tal, es necesario detener las operaciones normales de dicha planta. Petrobras tiene en total 15 refinerías con capacidad para refinar entre $31 \mathrm{mbd}$ y $365 \mathrm{mbd}$. Cuando prueba un catalizador, tiene diversas alternativas en refinerías con distinta capacidad instalada. Pemex, a su vez, cuenta con seis refinerías con capacidades de entre $185 \mathrm{mbd}$ y $330 \mathrm{mbd}$, con lo cual el costo de oportunidad de la prueba industrial implica perder un volumen importante de refinados. En Colombia existen sólo dos refinerías, cuya capacidad de refinación conjunta se encuentra entre $80 \mathrm{mbd}$ y $250 \mathrm{mbd}$, lo cual probablemente implica un costo de oportunidad distinto del caso de una prueba industrial en México.

\section{c) Comercialización del catalizador}

Si la prueba en la refinería es exitosa, entonces se realiza la producción y comercialización del catalizador a escala industrial, aunque las capacidades de producción de catalizadores de los tres países son significativamente diferentes. Petrobras tiene capacidad propia para probar y producir catalizadores a baja escala; la producción a mayor escala es a través de la Fábrica Carioca de Catalisadores, SA (FCCSA). Esta empresa fue resultado de la asociación entre Petrobras, Akzo Nobel y Oxiteno (1985), aunque hoy en día los únicos socios son Petrobras y Albermarle Catalysts, cada uno con la mitad del capital (Fábrica Carioca de Catalisadores, SA, s.f.). Actualmente, la FCCSA suministra a Petrobras el 95\% de los catalizadores requeridos para producir combustibles, mientras que el $5 \%$ restante lo producen empresas extranjeras (Albermarle, Avantium y Kobe Steel).

Colombia, por su parte, no cuenta con empresas productoras de catalizadores para refinar petróleo y tampoco existen empresas que los prueben industrialmente. Esto ha conducido a Ecopetrol a comprar sus catalizadores a compañías extranjeras (FCCSA, BASF, Grace Davison, Albermarle, Criterion y Haldor Topsoe). Sin embargo, dada su estrategia tecnológica de coproducir catalizadores, Ecopetrol ha desarrollado alianzas con Grace Davison, Albemarle y UOP. Estos catalizadores incorporan, como un aditivo, la trampa de vanadio, ${ }^{5}$ una mejora incremental producida por el ICP de Ecopetrol.

\footnotetext{
${ }^{5}$ Es un aditivo que captura dicho metal para preservar la actividad y selectividad de los catalizadores de craqueo catalítico. El vanadio, presente en las cargas de petróleo crudo, contamina y destruye los componentes del catalizador utilizado en el proceso, disminuyendo su actividad y los rendimientos de los productos derivados (entrevista a personal del área de Catálisis-Ecopetrol, agosto de 2009).
} 
En México, Pemex tiene limitaciones para realizar la prueba industrial de los catalizadores. Tampoco cuenta con una empresa nacional con capacidad para probarlos y producirlos, aunque se ha intentado en tres ocasiones crear empresas mexicanas para tal fin. El primer intento fue EXIMGRO (1981), la cual fabricaba un catalizador de HT desarrollado por el IMP. La empresa cerró cuando se vio afectada por las variaciones en la tasa de cambio, ya que utilizaba insumos extranjeros. El segundo intento fue PYOSA (1982), la cual fabricó los primeros catalizadores para el tratamiento de la gasolina y el gas LP. Sin embargo, debido al reducido tamaño del mercado de este tipo de catalizadores se extinguió esa línea de producción y con ella la empresa (Aboites et al., 2004). Finalmente, el último intento fue la Compañía Internacional de Catalizadores de Impregnación (Soria, 2004). Esta compañía se fundó con la participación del IMP y las empresas Protexa, United Catalysts Inc. y Quimi Corp Internacional. Sin embargo, el bajo nivel de rentabilidad debido a las dificultades para disminuir los precios de los insumos necesarios la hicieron inviable para producir catalizadores. Después de abandonar esta línea de producción, se convirtió en una simple proveedora de servicios para los catalizadores que Pemex Refinación recibía del IMP (Soria, 2004). Hubo más experiencias durante los noventa, cuando otras empresas (Almexa, Química Flúor, Cuproquim, Hexaquimia y Pennwalt) fabricaron lotes selectos de catalizadores de distintos tipos (ocho de los 33 tipos de catalizadores usados por Pemex Refinación).

Sin embargo, antes del fin de esa década también abandonaron la industria nacional (Gil, 1999). En efecto, esta falta de continuidad en el desarrollo de la industria y la tecnología propia denota, una vez más, que la verdadera política tecnológica de Pemex ha sido transferir la tecnología necesaria desde el exterior y contratar empresas extranjeras para tareas tecnológicas y productivas que no puede realizar.

En México, durante el periodo 1974-2001, el IMP logró alianzas tecnológicas con compañías extranjeras (UOP, UCI, Engelhard, Criterion, Intercat, Grace, Acreon, Katalco) coproduciendo 43 catalizadores para Pemex en ese lapso. El IMP diseñaba la formulación primaria y realizaba pruebas hasta el nivel de planta piloto. Con ese diseño tecnológico, la compañía extranjera realizaba una primera prueba industrial en refinerías de Estados Unidos (Aboites et al., 2004; Soria, 2004). Informantes calificados del IMP han asegurado que hasta el año 2010 algunos de estos catalizadores se seguían produciendo bajo este mismo esquema. Sin embargo, actualmente, la estrategia tecnológica de Pemex ha dado un giro hacia el mercado internacional. Ahora, cuando requiere un nuevo catalizador, realiza 
una licitación, en la que pueden participar el IMP en alianza con una compañía extranjera o las compañías extranjeras por su cuenta.

\section{Cuadro 3. Resumen de las capacidades tecnológicas desarrolladas en Brasil, Colombia y México, 2011}

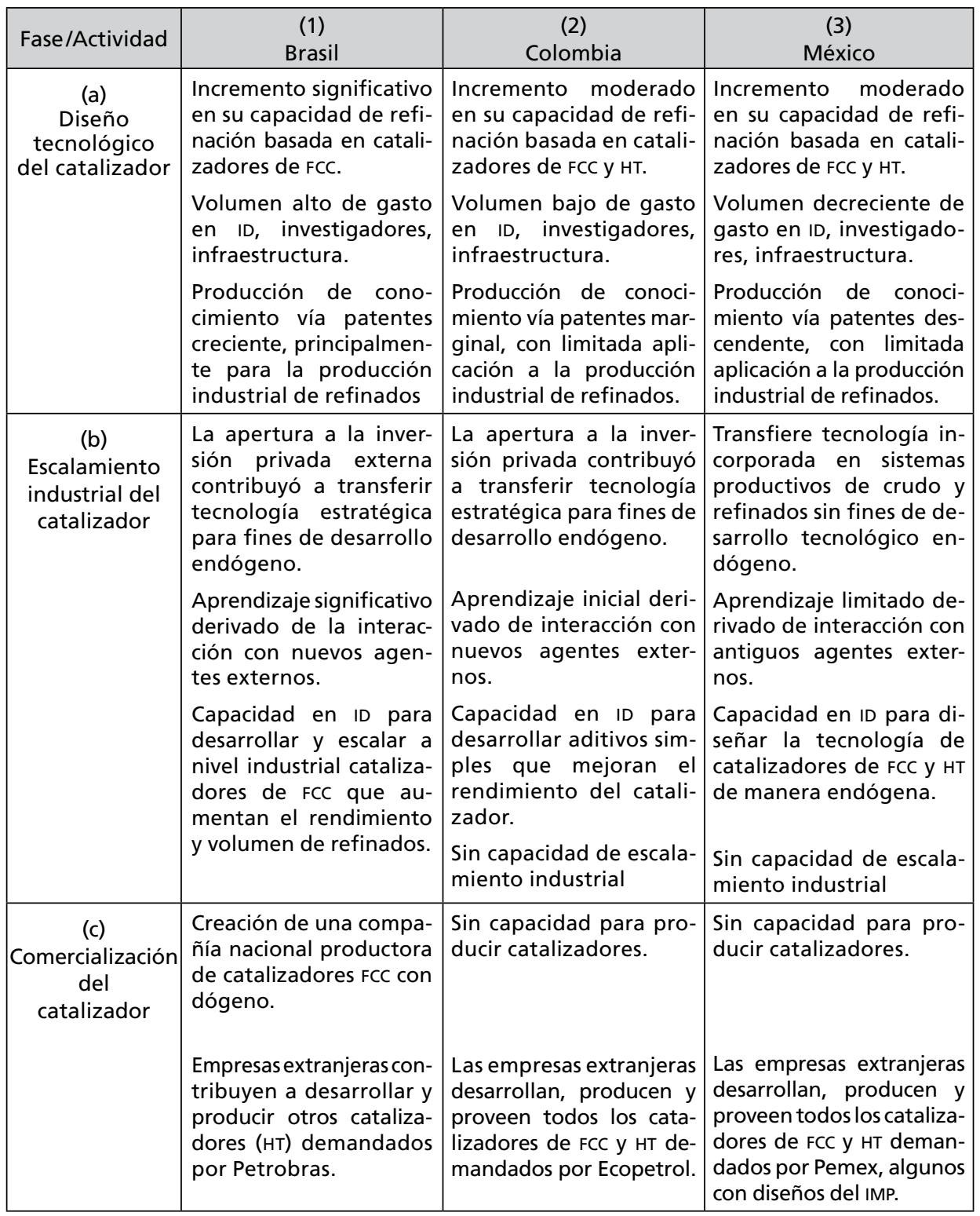

Fuente: Elaboración propia, a partir de Mora (2010) y Soria (2004). 
En la realidad, la mayor parte de estas licitaciones son ganadas por estas últimas (Shell, Standard Oil y ExxonMobil). Por último, en el caso de los tres países analizados, las compañías que suministran los catalizadores también son las encargadas de prestar los servicios de su mantenimiento en planta.

Un resumen de los principales elementos y características de las capacidades tecnológicas desarrolladas por la industria petrolera de cada uno de los países evaluados se presenta en el cuadro 3 .

\section{III. ¿CONVERgen O DIVERgen BRASIL, COLOMbia Y MÉXICO EN EL DESARROLLO DE CAPACIDAD TECNOLÓGICA DE CATALIZADORES?}

Las capacidades tecnológicas de los países seleccionados se examinan en esta sección distinguiendo las convergencias y divergencias en el desarrollo de capacidades para el diseño y producción de catalizadores. En efecto, conforme a la evolución de sus procesos de producción o transferencia de conocimiento tecnológico endógeno o exógeno en las distintas fases de desarrollo tecnológico y consumo de un catalizador en la industria de refinación del petróleo, las modalidades que adoptó la innovación incremental de los catalizadores (cuadro 4) en Brasil (modalidad C), Colombia (modalidad A) y México (modalidad B) son las que se exponen a continuación.

Cuadro 4. Modalidades de la innovación tecnológica de catalizadores en países en desarrollo

\begin{tabular}{|l|c|c|c|}
\hline \multirow{2}{*}{\multicolumn{2}{|c|}{ Fase de la innovación }} & \multicolumn{3}{c|}{ Modalidad de la innovación } \\
\cline { 2 - 4 } & A Baja & B Media & C Alta \\
\hline Fase I: Diseño tecnológico & Exógeno & Endógeno & Endógeno \\
\hline Fase II: Escalamiento industrial & Exógeno & Exógeno & Endógeno \\
\hline Fase III: Comercialización & Exógeno & Exógeno & Endógeno \\
\hline
\end{tabular}

Fuente: Elaboración propia.

\section{Modalidad A: las fases I, II y III son exógenas}

En Colombia, la demanda de catalizadores por parte de la empresa petrolera nacional depende casi completamente del mercado externo para ser satisfecha. Esto significa que en lo fundamental la fase de investigación y desarrollo es exógena $\mathrm{y}$ el conocimiento tecnológico se transfiere incorporado en los catalizadores 
importados (cuadro 3, columna 2, fila a). Lo anterior implica a su vez, que las fases de escalamiento, producción y venta industrial son exógenas, es decir, se concretan mediante una compañía extranjera productora y comercializadora de catalizadores (cuadro 3, columna 2, fila c). Sin embargo, en el caso de Colombia el grado endógeno de la producción de diseños tecnológicos de catalizadores no es nulo. Por una parte, esta modalidad exige una base de conocimiento tecnológico mínima para adaptar el producto a las refinerías nacionales (cuadro 3, columna 2, fila b). Por la otra, en la última década se dio un significativo y creciente esfuerzo en ID a través del ICP, con lo cual se han ido logrando algunos éxitos de comercialización industrial que podrían eventualmente encaminar a su industria petrolera hacia una modalidad B.

\section{Modalidad B: las fases II y III son exógenas}

En México existe capacidad tecnológica para que una porción de la demanda de catalizadores por parte de Pemex Refinación sea satisfecha conforme a diseños tecnológicos surgidos de la actividad de ID endógena realizada principalmente por el IMP (cuadro 3, columna 3, fila a). Sin embargo, dado que los esfuerzos de las últimas tres décadas para instalar una fábrica de catalizadores en México han fracasado, el diseño tecnológico del IMP sólo puede plasmarse en un catalizador fabricado por empresas extranjeras con capacidad para producir catalizadores a escala industrial (cuadro 3, columna 3, fila b). Con esto, la tercera fase también es de carácter exógeno y es realizada por la misma compañía extranjera productora del catalizador (cuadro 3, columna 3, fila c). Cabe señalar, sin embargo, que la fase de comercialización implica un proceso de servicios tecnológicos del catalizador en planta, los cuales son ofrecidos tanto por empresas extranjeras como por el IMP. Así, a diferencia de la modalidad A, en la modalidad B se produce conocimiento tecnológico propio, aunque sin tener todavía capacidad productiva y comercializadora propias.

En el caso del IMP es importante señalar una situación paradójica. Por una parte, entre los años setenta y noventa hubo esfuerzos sostenidos de Pemex y el IMP para desarrollar diseños tecnológicos de catalizadores propios que fueran más adecuados a sus plantas de refinación y a la demanda de productos con los que se cuenta en México. Por la otra, con esa misma vehemencia, durante la última década, tanto Pemex como el IMP no sólo han desistido de realizar nuevos proyectos de investigación para el desarrollo tecnológico endógeno, sino que también han ido erosionando (Aboites, et al., 2004) o destruyendo abiertamen- 
te (Soria, 2004) las capacidades tecnológicas en materia de catalizadores que se construyeron durante las décadas previas. Esto significa, en términos de este modelo, que la industria petrolera mexicana en vez de avanzar hacia la modalidad C de innovación, al parecer retrocede para estancarse en el estado inicial de la modalidad B.

\section{Modalidad C: las fases I, II y III son endógenas}

Finalmente, en el caso de Brasil todas las fases del proceso de producción de catalizadores se desarrollan de manera endógena, en el marco de una competencia oligopólica con empresas extranjeras, por lo que la empresa petrolera nacional si bien es dominante tampoco es la única fuente de tecnología para la industria de los hidrcarburos brasileña. En el caso de la refinación de gasolina, cuando Petrobras demanda un nuevo catalizador de FCC, el Cenpes tiene la capacidad de ID para producir la tecnología (cuadro 3, columna 1, fila a). Posteriormente, la compañía nacional productora de catalizadores puede escalar dicha tecnología, realizar la prueba y producirlo para consumo a escala industrial (cuadro 3, columna 1, filas b y c).

En el caso de Brasil, puede decirse que los cambios institucionales de los años noventa fueron definitorios en torno a la condición monopólica de la empresa petrolera nacional. En efecto, la estructura organizativa de la industria del petróleo brasileña se modificó al transformarse de un monopolio estatal en una empresa dominante de capital mixto en un contexto regional y global de competencia oligopólica. Este cambio abrió, entre otras cosas, las puertas del aprendizaje por interacción con nuevos agentes y condujo a Petrobras hacia un proceso de desarrollo tecnológico endógeno mediante alianzas con capital privado extranjero. Con esto, aumentaron la capacidad productiva de refinación de combustibles y el desempeño económico de la industria.

En resumen, el presente artículo establece entonces que en términos de la innovación tecnológica de catalizadores para refinar petróleo en países en desarrollo, la mayoría de los países que cuentan con una industria de refinación de petróleo se encuentran en un principio operando bajo la modalidad A (cuadro 3, columna 2) y conforme van desarrollando capacidad tecnológica y productiva endógena se acercan cada vez más a la modalidad C (cuadro 3, columna 1), cuando todo el proceso de un catalizador se puede realizar de manera propia. Entonces, el grado endógeno de producción de catalizadores se refiere al punto en el 
cual se encuentra la industria petrolera de un país dado, al transitar de la modalidad A, a través de la B (cuadro 3, columna 3), hacia la modalidad C.

\section{HALLAZGOS Y REFLEXIONES FINALES}

La industria petrolera latinoamericana ha transitado por diferentes fases de desarrollo económico y social, empezando por el periodo de industrialización sustitutiva de la primera mitad del siglo XX -cuando el Estado de cada país analizado recuperó el control de su industria petrolera-; luego, a través de la grave crisis económica de los ochenta que profundizó la brecha en la distribución del ingreso en América Latina, hasta la actualidad, cuando en una parte significativa de los países con industria petrolera el Estado abrió la inversión en este sector al capital privado nacional y extranjero y eliminó el monopolio estatal absoluto, favoreciendo así la competencia oligopólica. En este tránsito, han sido los cambios institucionales y las estrategias tecnológicas iniciadas desde los años noventa lo que ha marcado las principales diferencias en el desempeño económico de la industria del petróleo de los países aquí analizados, durante las últimas dos décadas. La evidencia permitió constatar que estas tres naciones latinoamericanas en desarrollo no han sido simples receptoras de tecnología de los países desarrollados. En distinto grado han construido procesos endógenos de aprendizaje y construcción de capacidad tecnológica en su industria petrolera. En particular, cada una ha seguido determinada modalidad de innovación tecnológica reflejada en las estrategias de diseño, producción y comercialización de catalizadores para refinar combustibles del petróleo.

El principal hallazgo es: 1) Un significativo avance de Brasil de la modalidad B a la C, 2) un progreso de Colombia que pronto la podría llevar de la modalidad A hasta la B, y 3 ) un repliegue en México, cuya capacidad tecnológica acumulada en materia de catalizadores se encuentra en retroceso de la modalidad B hacia la A.

Brasil construyó, desarrolló y acumuló capacidad tecnológica exitosamente, alcanzando en la última década una modalidad de innovación tecnológica de catalizadores con mayor grado endógeno (C) que México o Colombia. En parte, esto ha sido un resultado de los cambios institucionales en la industria del petróleo desde la década de los noventa, que fomentaron la inversión y la competencia, al transformar el monopolio estatal en un oligopolio con la presencia de capital privado nacional y extranjero. Con ello, también se favoreció tanto la transferencia de tecnología como la construcción y desarrollo de capacidad tec- 
nológica propia. Ésta se constata en el alto nivel de gasto en ID, en el número de investigadores de la empresa petrolera nacional y, particularmente, de los dedicados a desarrollar catalizadores, así como en la infraestructura para ID y en la capacidad para producir conocimiento tecnológico medido por patentes. Asimismo se observa en el incremento de su capacidad para producir crudo y refinados en la última década, y en la disminución de su déficit de comercio exterior de estos productos. En suma, las industrias petrolera y de catalizadores de Brasil sobresalen entre los tres países analizados como las de mayor dinamismo institucional, tecnológico y económico en la última década.

Colombia muestra un menor grado endógeno que Brasil y México en su modalidad de innovación tecnológica (A) de catalizadores, debido al menor tamaño de la industria y lo reciente de sus esfuerzos por desarrollar capacidad tecnológica. Sin embargo, al igual que Brasil, avanza a partir de los cambios institucionales en el sector que sustituyeron el monopolio absoluto del Estado por una competencia oligopólica con empresas extranjeras. Con ello, no sólo se ha fomentado una mayor inversión en general, sino, en particular, se ha incrementado la inversión en ID tecnológica en la industria. En efecto, se ha desarrollado capacidad tecnológica doméstica para diseñar aditivos que mejoran el rendimiento de los catalizadores existentes en el mercado colombiano, mediante procesos de aprendizaje ligados a alianzas tecnológicas con las empresas extranjeras que los producen. En el plano productivo, en conjunto, estos cambios han contribuido a elevar la producción de petróleo y la capacidad de refinación, reducir la exportación de crudo y aumentar el superávit comercial de refinados en la región.

México mantiene una situación divergente con los dos casos anteriores. En primer lugar, el cambio institucional en el sector del petróleo del tipo aplicado en Brasil y Colombia ha sido nulo. Por el contrario, las actuales reglas sobre inversión pública no le permiten a Pemex crecer y desarrollarse como las demás empresas petroleras en América Latina. Su estructura histórica de inversión y producción a nivel latinoamericano se mantiene, pero como un gran exportador de petróleo que refina en combustibles sólo la mitad de su producción de crudo e importa el grueso de los petroquímicos requeridos por la economía. En segundo lugar, como empresa petrolera no cuenta con un centro propio de ID. Más bien, ha apoyado y financiado ciertas actividades de ID a lo largo de la evolución del IMP. En particular, con el financiamiento y colaboración de Pemex, en el área de catalizadores del IMP se logró desarrollar a lo largo de las últimas cuatro décadas capacidad tecnológica para diseñar catalizadores a la medida de las plantas de Pemex Refinación. Sin embargo, a diferencia de Brasil y luego de tres 
intentos infructuosos por conformar una empresa mexicana productora y comercializadora de catalizadores, Pemex Refinación requiere de una alianza con las empresas extranjeras para producir y comercializar catalizadores. Con ello, predomina la modalidad de innovación B en el grado endógeno del proceso de diseño, producción y comercialización de catalizadores para refinar combustibles. Finalmente, la evidencia reciente sobre la capacidad del IMP para producir conocimiento tecnológico sobre catalizadores, medida por gasto en ID, capital humano y patentes, indica que las estrategias tecnológicas de este instituto no necesariamente empatan con las de Pemex, lo que causa un deterioro de la capacidad tecnológica acumulada por el IMP en el área de catalizadores y, por ende, de la industria del petróleo en México.

\section{REFERENCIAS BIBLIOGRÁFICAS}

Aboites, Jaime, et al. (2004), La triada innovadora, México, Editorial Siglo XXI. Abramovitz, Moses (1956), "Resource and Output Trends in the United States Since 1870", American Economic Review, Papers and proceedings, 46(2), p5-23.

Bell, Martin (1984), “ 'Learning' and the Accumulation of Industrial Technological Capacity in Developing Countries”, en K. King, K., y Fransman, M. (eds.), Technological Capacity in the Third World, London, Macmillan, pp. 187-209.

_ , y Pavitt, Keith (1995), "The Development of Technological Capabilities", en Haque, I. (ed.), Trade, Technology and International Competitiveness, Washington, The World Bank, pp. 69-101.

Campbell, J., y Pedersen, K. (2001), The Rise of Neoliberalism and Institutional Analysis, USA, Princeton University Press.

Cohen, Wesley, y Levinthal, Daniel (1989), "Innovation and Learning: the Two Faces of R\&D”, The Economic Journal, No. 99, September, pp. 569-596.

CONACYT (s.f.), información sin título. Recuperada de www.conacyt.mx. Consultada en agosto de 2010.

Dahlman, Carl, y Westphal, Larry (1982), “Technological Effort in Industrial Development. An Interpretative Survey of Recent Research", en Stewart, F., y James, J. (eds.), The Economics of New Technology in Developing Countries, London, Frances Pinter.

Dosi, Giovanni (1981), Technical change and survival: Europe's semiconductor industry, Brighton, Sussex European Research Centre/University of Sussex.

- (1988), "The nature of the innovation process", en Dosi, G., et al., Technical Change and Economic Theory, cap. 10 "Technical Change and Economic 
Theory”, Pisa, Italy, LEM Book Series, Laboratory of Economics and Management, Sant'Anna School of Advanced Studies.

Ecopetrol (2008a), Estados contables 2006 y 2007, Colombia, Empresa Colombiana de Petróleos.

- (2008b) Conferencia de inversiones- BID, Colombia, Empresa Colombiana de Petróleos.

(2008c), Histórico de las cifras volumétricas operativas de Ecopetrol 2000-2007, Colombia, Empresa Colombiana de Petróleos.

- (2009), Informe Anual 2008, Colombia, Empresa Colombiana de Petróleos..

_ (2010a), Informe Anual 2009, Colombia, Empresa Colombiana de Petróleos.

- (2010b), Resultados financieros no auditados, para el primer trimestre de 2010, Colombia, Empresa Colombiana de Petróleos.

- (s.f.), información sin título. Recuperada de: www.ecopetrol.com.co. Consultada en mayo de 2010.

EIA (s.f.), US Energy Information Administration. Recuperado de: www.eia.gov. Consultado en septiembre de 2011.

Fábrica Carioca de Catalisadores, SA (s.f.), información sin título. Recuperada de: www. fccsa.com.br. Consultada en junio de 2010.

Figueiredo, Paulo (2002), "Learning processes features and technological capability accumulation: explaining inter-firm differences", Technovation, 22, pp. 685-698.

Fransman, Martin (1986), "International competitiveness, technical change and the state: The machine tool industry in Taiwan and Japan", World Development. Elsevier, Vol. 14(12), pp. 1375-1396.

Garay, Luis (1998), Colombia: estructura industrial e internacionalización 1967-1996, s.l., Universidad Nacional de Colombia.

Gil, Jorge (1999), "Estructuración de una comunidad científica: el caso de la catálisis", tesis de Maestría en Economía y Gestión del Cambio Tecnológico, México, UAM-Xochimilco.

Herbert-Copley, Brent (1990), "Technical Change in Latin American Manufacturing Firms: Review and Synthesis", World Development, Vol. 18. No. 11, pp. 1457-1469.

Instituto Mexicano del Petróleo (2008a), Seminario: Oportunidades de creación de conocimiento para el futuro energético de México, México. (2008b), Consolidado de indicadores operativos 2008, México.

Katz, Jorge (1976), Importación de tecnología, aprendizaje e industrialización dependiente, México, FCE.

_ (1987), "Domestic technology generation in LDCs: a review of research findings", 
en Katz, J. (ed.), Technology Generation in Latin American Manufacturing Industries, London, Macmillan.

(2007), "Cambios estructurales y ciclos de destrucción y creación de capacidades productivas y tecnológicas en América latina" (documento de discusión).

- (2008), Una nueva visita a la teoría del desarrollo económico, Chile, CEPAL, Colección Documentos de Proyectos.

Kuznets, Simon (1966), Modern Economic Growth. Rate, Structure and Spread, New Haven, Yale University Press.

Lall, Sanjay (1991), "Explaining industrial success in the developing world", en Balasubramanyam, V. N., y Lall, S. (eds.), Current Issues in Development Economics, London, pp. 118-155.

_ (1993), “Technological Capabilities", en Salomon, J.J. (ed.), The Uncertain Question: Science, Technology and Development, Tokyo, United Nations University Press, pp. 264-301.

Lundvall, Bengt (1992), National Systems of Innovation. Towards a Theory of Innovation and Interactive Learning, London, Pinter Publishers.

Mora, H. (2010), Comparación de las capacidades tecnológicas de Brasil, Colombia y México en la producción de catalizadores para refinar petróleo, tesis de maestría en Economía y Gestión de la Innovación, México.

Nelson, Richard (1981), "Research on Productivity Growth and Productivity Differences: Dead ends and departures", Journal of Economic Literature, Vol. 19, September, pp. 1029-1064.

- y Winter, Sidney (1982), An evolutionary theory of Economic Change, Cambridge, Ma., Belknap Press of Harvard University Press.

North, D. (1993), Instituciones, cambio institucional y desempeño económico, México, FCE.

Pavitt, Keith (1982), “R\&D, patenting and innovative activities: A statistical exploration”. Research Policy, Elsevier, Vol. 11 (1), pp. 33-51.

Pemex (2007), Base de datos institucional sobre refinerías, México, Petróleos Mexicanos. (2009), Informe anual 2008, México, Petróleos Mexicanos.

— (s.f.a), "Historia de Petróleos Mexicanos". Recuperado de: www.pemex.com. Consultado en mayo de 2010.

_ (s.f.b), "Inversión de Pemex". Recuperado de: www.pemex.com. Consultado en mayo de 2010.

Petrobras (2007), Plan de negocios 2008-2012, Brasil, Petróleos Brasileños.

— (2009 a), Informe anual 2008, Brasil, Petróleos Brasileños.

— (2009 b), Resultados del ejercicio 2008, Brasil, Petróleos Brasileños. 
- (2010), Resultados del ejercicio 2009, Brasil, Petróleos Brasileños.

- (s.f.), información sin título. Recuperado de: www.petrobras.com. Consultado en mayo de 2010.

Rath, Amitav (1990), "Science, technology, and policy in the periphery: A perspective from the center", World Development, Vol. 18, No. 11, pp. 1429-1443.

Sener (2007 a), Prospectiva del mercado de petróleo crudo 2007-2016, México, Secretaría de Energía.

— (2007b), Prospectiva de petroliferos 2007-2016, México, Secretaría de Energía.

- (2008), Refinación, la industria en México, México, Secretaría de Energía.

Soria, Manuel (2004), "La organización de la innovación tecnológica: Los catalizadores del Instituto Mexicano del Petróleo", tesis de Doctorado en Estudios Organizacionales, UAM-Iztapalapa, México.

Teitel, Simon (1984), "La creación de tecnología en las economías semiindustrializadas", en Teitel, S., y Westphal, L., Cambio tecnológico y desarrollo industrial, México, FCE.

Torres, Arturo (2006), “Aprendizaje y construcción de capacidades tecnológicas”, Journal of Technology, Management \& Innovation, Vol. 1, No. 5, pp. 12-24.

Vernon, Raymond (1989), “Technological development: The historical experience”, Seminar Paper No. 39, Economic Development Institute/World Bank. 